\title{
Radial velocities and spectrophotometric properties of southern binary galaxies ${ }^{\star}$
}

\author{
R.E. de Souza ${ }^{1}$, D.F. de Mello ${ }^{2,3}$, and S. dos Anjos ${ }^{1}$ \\ 1 Astronomy Department, University of São Paulo, C.Postal 9638, SP 01065-970, Brazil \\ 2 Observatório Nacional-DAN, C.Postal 23002, RJ 20921-400, Brazil \\ 3 Visiting Astronomer, Cerro Tololo Inter-American Observatory
}

Received September 23, 1996; accepted January 10, 1997

\begin{abstract}
We present spectrophotometry of a sample of 26 Southern binary galaxies 17 pairs were confirmed to be physically associated with typical separation of $136 \mathrm{~h}^{-1} \mathrm{kpc}$. Pairs with velocity difference $\Delta v \geq$ $600 \mathrm{~km} \mathrm{~s}^{-1}$ are probably not physically bound. A comparison with field galaxies suggests that galaxies in pairs tend to have a younger population probably stimulated by tidal interaction. No correlation between the young population and projected separation was found.
\end{abstract}

Key words: galaxies: interactions - galaxies: stellar content

\section{Introduction}

The study of binary galaxies is one the first steps towards understanding the physics of interaction between galaxies. Many works have been done in the past few years in order to understand the subtleties resulting from the gravitational interaction between pairs of galaxies. It is likely that the majority of these systems have approximately circular orbits with mean eccentricity $e \simeq 0.25$. Therefore, due to the dynamical friction they should merge on a time scale of the order of $0.2 H_{0}^{-1}$ (Karachentsev 1990). We remark, however, that these results rests on a sample affect by selection biases, due to the isolation criteria, and an oversimplified treatment of dynamical friction. On the other hand it is well known that gravitational interaction stimulates star formation. For instance, tidal distortions in interacting pairs could contribute up to $25-60 \%$ of the galaxy luminosity (Schombert et al. 1990). Moreover, the mean star formation rate in physical pairs, measured from

Send offprint requests to: R.E. de Souza

* Table 1 is also available at the CDS via anonymous ftp to cdsarc.u-strasbg.fr (130 79128 5) or via http://cdsweb.ustrasbg.fr/Abstract.html
$\mathrm{H} \alpha$ emission, can be three times larger than the observed rate on non-interacting systems (see Kennicutt 1990 and references therein). More recently CO observations suggest that more gas is available in paired galaxies, resulting that the star formation is enhanced in these systems (Combes et al. 1994). Reduzzi \& Rampazzo (1996) found that even a moderate interaction is able to constrain the gas into nuclear regions and enhance the star formation and nuclear activities in binary galaxies.

From the dynamical point of view, binary galaxies present several indications of the damage caused by the interaction. In the case of disk systems the classical work by Toomre \& Toomre (1972) pointed out very clearly that the interactions during direct encounters are far more efficient than the retrograde orbits. Moreover, direct encounters tend to form two spiral arms, while a single arm is favored on retrograde encounters (Athanassoula 1990). Also, the formation of bars tend to be stimulated; $81 \%$ of the binary galaxies are barred, while only $63 \%$ of the spirals are barred in the general field. The dynamical interaction also affects the interstellar medium of the objects favoring a more fragmented distribution of clouds. As a result the star formation rate is temporarily enhanced, reaching a maximum approximately $10^{8}$ years after the point of minimum separation.

In order to address some of these issues we begun a program to study binary galaxies in the Southern hemisphere. As a first step we have built a sample of isolated pairs extracted from the ESO-LV catalog (Soares et al. 1995). Statistical properties of this sample shows that the luminosity function is very similar to the one found for a sample extracted from the list of Karachentsev (1985) for the Northern hemisphere (Xu \& Sulentic 1991). Moreover, the $V / V_{\text {MAX }}$ test shows that our list is complete for the pairs having a primary component brighter than $B_{\mathrm{T}}=13.5$.

However, several objects in our list do not have radial velocity measurements. Actually, out of 189 pairs only $30 \%$ of them have radial velocity published in the literature. Therefore, we began an observational program in 
order to complete the missing radial velocities, since this information is crucial in order to better understand the properties of this sample. In this paper we present the first results of these observations.

\section{Data acquisition}

Long-slit spectra were taken at the CTIO $1.5 \mathrm{~m}$ telescope in February 1996. We used a Loral $1200 \times 800$ CCD $(1$ pixel $=15 \mu \mathrm{m})$, grating \#13 with $150 \mathrm{l} \mathrm{mm}^{-1}$ centered on $5000 \AA$ in first order. In this configuration the wavelength range covers from $\approx 3000 \AA$ to $8500 \AA$ with a reciprocal dispersion of $5.6 \AA /$ pix. The CCD gain was fixed on $2.05 \mathrm{e}^{-} / \mathrm{ADU}$ and a corresponding readout noise of $6.5 \mathrm{e}^{-}$. During the galaxy observations the slit was fixed on 130 microns resulting in a final spectral resolution of $14.4 \AA$. Wavelength calibration was done with He-Ar comparison lamp. However, since the CCD sensitivity drops quite abruptly in the blue we needed to make two separated calibration exposures. A deep calibration was adopted for the extreme blue with $t_{\exp }=30^{\mathrm{s}}$. For the red a normal exposure of of $2^{\mathrm{s}}$ was sufficient to obtain good calibration lines.

Typical exposure time for our objects were three observations of $900^{\mathrm{s}}$ in order to be able to remove cosmic rays more easily. We have also observed a sample of late type stars for radial velocity calibration and CTIO spectrophotometric standard stars (Baldwin \& Stone 1984). In Table 1 we present a list of observed galaxies with the same identification code adopted by Soares et al. (1995).

Standard data reduction techniques were followed using the IRAF package. The extraction was done with apall task in each one of the spectra and later on combined to a single spectra removing the eventual presence of cosmic rays. We used the optimal extraction algorithm (Horne 1986) with weights computed from the estimated noise. The tracing and sky subtraction of each object was interactively determined for each image. The extracted spectra were wavelength calibrated and rebinned to a linear scale of $5 \AA$ in the range $3700-8520 \AA$. A correction for sensitivity was also applied adopting the average determination on the 5 observed nights using our spectrophotometric observations and the mean extinction coefficient for CTIO.

The radial velocities were determined from the cross correlation package of IRAF, using standard velocity stars as templates. In objects with strong emission lines we have eliminated these regions before applying the correlation technique on the absorption lines. Besides the standard stars, we have also observed a sample of 12 galaxies with known radial velocities. A comparison of our measurements with the literature resulted that the mean RMS error on our determination is of the order of $35 \mathrm{~km} \mathrm{~s}^{-1}$. Moreover, we have also detected a systematic drift of $26 \mathrm{~km} \mathrm{~s}^{-1}$ which was removed from our velocities. From those objects with emission lines we have also determined the emission velocities and the two determinations are consistent to within an error of $42 \mathrm{~km} \mathrm{~s}^{-1}$. Our results are shown in Table 1. In the first two columns we present the object identification as it is described in Soares et al. (1995) and the ESO identification code. In Cols. 3 and 4 we present the total blue magnitudes and the morphological type extracted from the ESO-LV catalog (Lauberts \& Valentijn 1989). The absorption line velocities and estimated error $(\delta v)$ are presented in Cols. 5 and 6 . In Cols. 7 and 8 we present the $R$ quality parameter of Tonry \& Davis (1979) and the peak value of the correlation spectra. For a few objects we were able to determine the emission line velocities (Cols. 9 and 10) based on the number of observed lines (Col. 11). In Col. 12 we have the radial velocities of the objects collected from the ZCAT redshift catalog (Davis \& Huchra 1982). In Col. 13 we show the mean residual parameter in the blue, that will be defined in Sect. 3 bellow. In the last two columns we present the projected separation in $\mathrm{kpc}$ and the velocity difference in $\mathrm{km} \mathrm{s}^{-1}$ using $h=1$.

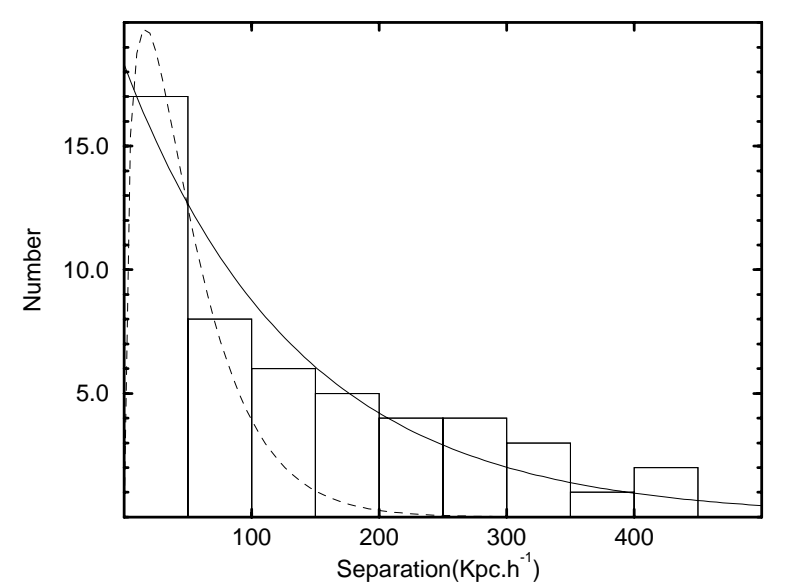

Fig. 1. The projected pair separation distribution showing that our sample (solid line) have a larger contribution of loose pairs then the Karachentsev sample (dashed line)

\section{Discussion}

Using the present set of observations we were able to confirm the binary association of 17 out of 26 pairs. Approximately $30 \%$ of the original sample are found to be false pairs. Adding these new observations with the ones already existing in the literature we have 50 pairs of the list of Soares et al. (1995) with known radial velocities. In Fig. 1 we present an histogram of these objects as a function of the projected separation. The dashed line represents the distribution found on Karachentsev's sample of 487 systems. We remark that due to the isolation criteria the sample of Karachentsev (1987) is strongly 
Table 1. Radial velocity data

\begin{tabular}{|c|c|c|c|c|c|c|c|c|c|c|c|c|c|c|}
\hline$\overline{\mathrm{Obj}}$ & ESO & $B_{\mathrm{T}}$ & $T$ & $v_{\mathrm{abs}}$ & $\delta v$ & $R$ & $\max$ & $v_{\mathrm{ems}}$ & $\delta v$ & $\mathrm{nl}$ & $v_{\text {lit }}$ & $R_{40}$ & Sep & $\Delta V$ \\
\hline g222b & $250-0180$ & 15.1 & 3 & 4639 & 20 & 8 & 0.49 & 4682 & 84 & 3 & & 0.36 & 109 & 141 \\
\hline g238b & $158-0060$ & 15.3 & 0 & 11769 & 11 & 12 & 0.75 & 11950 & 5 & 2 & & 0.09 & - & - \\
\hline g240a & $203-0070$ & 14.3 & -3 & 5376 & 10 & 15 & 0.85 & & & & & 0.08 & 334 & 1060 \\
\hline g240b & $203-0040$ & 14.5 & 4 & 4316 & 14 & 10 & 0.70 & 4345 & 60 & 3 & & 0.46 & 334 & 1060 \\
\hline g255b & $486-0180$ & 15.6 & 1 & 12563 & 15 & 9 & 0.70 & & & & & 0.06 & 423 & 123 \\
\hline g266b & $158-0200$ & 15.4 & 3 & 10304 & 12 & 8 & 0.60 & & & & & 0.19 & 376 & 459 \\
\hline g266bc & $158-0201$ & 15.5 & 1 & 9845 & 13 & 13 & 0.74 & & & & & 0.14 & 376 & 459 \\
\hline g267a & $486-0380$ & 14.6 & -3 & 4156 & 8 & 15 & 0.85 & & & & & 0.08 & - & - \\
\hline g276a & $305-0220$ & 14.6 & 3 & 4627 & 9 & 6 & 0.52 & 4640 & 38 & 6 & & 0.87 & - & - \\
\hline g276b & $305-0310$ & 14.6 & 4 & 15114 & 9 & 8 & 0.71 & & & & & 0.08 & - & - \\
\hline g277b & $305-0230$ & 15.4 & 1 & 11717 & 14 & 12 & 0.72 & & & & & 0.07 & 448 & 156 \\
\hline g277bc & $305-0231$ & 16.4 & 6 & 11561 & 15 & 12 & 0.76 & & & & & 0.08 & 448 & 156 \\
\hline g278a & $253-0040$ & 13.4 & -5 & 4339 & 7 & 12 & 0.82 & & & & 4224 & 0.05 & 209 & 41 \\
\hline g278b & $253-0010$ & 14.7 & 7 & 4380 & 38 & 6 & 0.33 & & & & & 0.51 & 209 & 41 \\
\hline g280a & $306-0030$ & 14.4 & 4 & 4687 & 0 & 11 & 0.66 & 4613 & - & 2 & & 0.38 & - & - \\
\hline g280b & $306-0011$ & 15.4 & 1 & 11138 & 10 & 13 & 0.66 & 11022 & 86 & 2 & & 0.07 & 494 & 83 \\
\hline g280bc & $306-0010$ & 15.5 & 1 & 11055 & 10 & 10 & 0.63 & 11084 & 39 & 3 & & 0.32 & 494 & 83 \\
\hline g281a & $204-0200$ & 14.6 & 5 & 10347 & 15 & 10 & 0.65 & & & & 10341 & 0.16 & - & - \\
\hline g281b & $204-0190$ & 14.8 & 6 & 4503 & 12 & 6 & 0.39 & 4706 & 66 & 3 & 4523 & 0.50 & - & - \\
\hline g284a & $568-0090$ & 14.3 & 6 & 3007 & 13 & 11 & 0.63 & 2724 & - & 1 & & 0.27 & - & - \\
\hline g284b & $568-0080$ & 14.7 & -5 & 5577 & 11 & 11 & 0.82 & & & & & 0.02 & - & - \\
\hline g304a & $305-0150$ & 14.0 & -4 & 7467 & 11 & 8 & 0.79 & & & & 7541 & -0.01 & 263 & 289 \\
\hline g304b & $505-0170$ & 15.8 & 0 & 7756 & 11 & 10 & 0.78 & & & & & 0.01 & 263 & 289 \\
\hline g307a & $506-0010$ & 13.3 & -4 & 3297 & 9 & 14 & 0.86 & & & & 3275 & 0.06 & 95 & 33 \\
\hline g318b & $443-0220$ & 16.0 & 8 & 3531 & 40 & 5 & 0.40 & 3433 & - & 2 & & 0.52 & 93 & 277 \\
\hline g323a & $443-0500$ & 14.5 & -2 & 5664 & 11 & 10 & 0.83 & & & & & 0.03 & 44 & 230 \\
\hline g323b & $443-0510$ & 15.6 & 3 & 5434 & 16 & 9 & 0.61 & 5185 & 80 & & & 0.19 & 44 & 230 \\
\hline g331a & $444-0101$ & 14.3 & 1 & 9304 & 10 & 10 & 0.78 & & & & & 0.07 & 23 & 53 \\
\hline g331b & $444-0100$ & 15.4 & 6 & 8986 & 38 & 8 & 0.42 & 9039 & 120 & 2 & & 0.22 & 23 & 53 \\
\hline g340a & $576-0760$ & 13.9 & -4 & 1695 & 9 & 15 & 0.86 & & & & & 0.04 & - & - \\
\hline g340b & $576-0730$ & 15.1 & 6 & 5561 & 57 & 8 & 0.51 & 5533 & 39 & 4 & & 0.50 & - & - \\
\hline g344a & $444-0760$ & 14.4 & -1 & 4273 & 9 & 15 & 0.85 & & & & & 0.08 & 36 & 426 \\
\hline g350a & $509-1010$ & 14.3 & -1 & 6805 & 9 & 9 & 0.79 & & & & 6827 & 0.03 & 160 & 238 \\
\hline g351a & $445-0510$ & 14.6 & -1 & 5000 & 10 & 14 & 0.78 & 5050 & 72 & 2 & 4995 & 0.13 & 169 & 321 \\
\hline g351b & $445-0480$ & 15.6 & 7 & 4679 & 26 & 9 & 0.48 & 4686 & 117 & 3 & & 0.40 & 169 & 321 \\
\hline g357b & $510-0550$ & 15.7 & 3 & 6167 & 12 & 10 & 0.66 & 6111 & 43 & 6 & & 0.18 & - & - \\
\hline g361b & $578-0260$ & 15.7 & 6 & 10891 & 16 & 9 & 0.74 & & & & & 0.00 & - & - \\
\hline g362a & $578-0290$ & 14.3 & -5 & 8720 & 11 & 12 & 0.82 & & & & & 0.05 & 287 & 19 \\
\hline g362ac & & & & 8950 & 12 & 9 & 0.27 & & & & & 0.02 & 287 & 19 \\
\hline g362b & $578-0320$ & 15.1 & 2 & 8701 & 11 & 10 & 0.80 & & & & & 0.03 & 287 & 19 \\
\hline g363a & $511-0180$ & 14.5 & 3 & 6395 & 10 & 13 & 0.71 & 6337 & 31 & 2 & & 0.24 & 43 & 14 \\
\hline g363b & $511-0200$ & 15.6 & 3 & 6381 & 14 & 12 & 0.63 & 6461 & 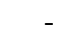 & 1 & & 0.14 & 43 & 14 \\
\hline g373a & $580-0430$ & 13.8 & -3 & 5990 & 11 & 10 & 0.79 & & & & 6054 & 0.23 & - & - \\
\hline g373b & $580-0410$ & 14.3 & 3 & 2954 & 23 & 6 & 0.48 & 3004 & 58 & 3 & & 0.36 & - & - \\
\hline g375a & $581-0240$ & 13.7 & 7 & 2422 & 51 & 5 & 0.30 & & & & 2279 & 0.27 & - & - \\
\hline g375b & $581-0220$ & 13.7 & 1 & 4264 & 9 & 13 & 0.83 & & & & 4266 & 0.05 & - & - \\
\hline n1741 & & 13.8 & $\mathrm{P}$ & & & & & 4136 & 16 & 10 & & 2.40 & - & - \\
\hline n3819 & & & & 6274 & 11 & 13 & 0.84 & & & & & 0.03 & - & - \\
\hline n3557 & $337-0160$ & 11.5 & -5 & 2917 & 10 & 13 & 0.85 & & & & 3151 & 0.03 & - & - \\
\hline n3923 & $440-0170$ & 10.6 & -3 & 1788 & 11 & 11 & 0.84 & & & & 1788 & - & - & . \\
\hline n4273 & & 12.0 & 5 & 2415 & 17 & 7 & 0.60 & 2492 & 29 & 3 & 2386 & 0.62 & - & - \\
\hline u4703a1 & & & & & & & & 3573 & 46 & 9 & & 0.86 & - & - \\
\hline $\mathrm{u} 4703 \mathrm{a} 2$ & & & & 3536 & 23 & 4 & 0.36 & 3580 & 34 & 6 & & & - & - \\
\hline
\end{tabular}

populated by tightly bound systems. In fact, he estimates that about $90 \%$ of the pairs with separation larger than $100 \mathrm{kpc}$ are omitted by his isolation criteria (Karachentsev 1990). On the other hand the isolation criteria adopted in Soares et al. (1995) takes into account the local density enhancement. Therefore, this sample includes a larger fraction of loose pairs, at the expense however of a larger contamination of false pairs. Actually the pairs in our sample are well described by an exponential distribution with a characteristic mean separation of $136 \mathrm{~h}^{-1} \mathrm{kpc}$ (solid line in Fig. 1). This value can be compared with the mean separation of $62 \mathrm{~h}^{-1} \mathrm{kpc}$ found on Karachentsev's sample, after corrections to evaluate only the isolated pairs.

In Fig. 2 we present the velocity difference distribution for our sample. The solid line represents the relation found for our sample, while the dashed line stands for the relation valid for Karachentsev's sample normalized for the number of objects in our sample. We can see that the 


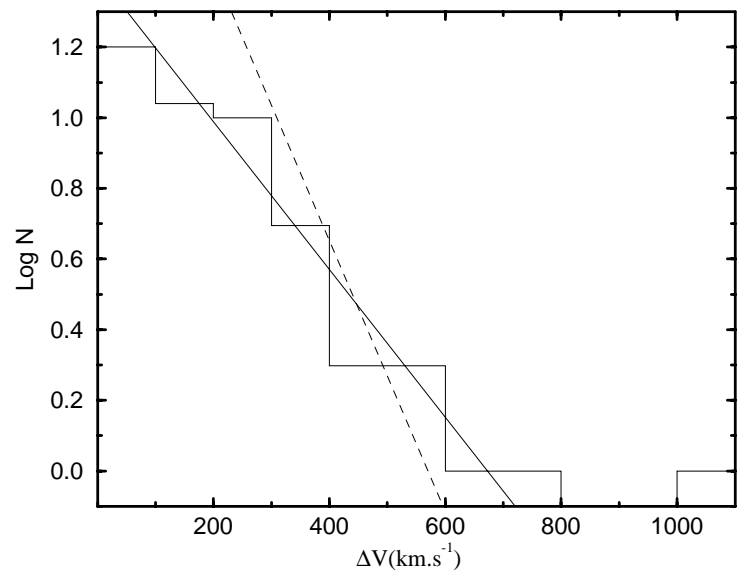

Fig. 2. The velocity difference distribution is well described by a linear relation (continous line) similar to the one found on Karachentsev's sample (dashed line)

velocity distribution of both sample are quite similar and it also agrees with Reduzzi \& Rampazzo (1995). The differences in the angular coefficient is probably related to the fact that our sample is richer in wide separtion pairs. Nevertheless we can infer that pairs with high velocity difference $\left(\Delta v \geq 600 \mathrm{~km} \mathrm{~s}^{-1}\right)$ are quite probably not bound and should be considered as false pairs as also suggested in previous work by Chengalur et al. (1993).

Since our spectra were flux calibrated we could also compare the shape of the spectra of binaries and field galaxies. For comparison we observed the elliptical galaxies NGC 3923 and NGC 3557 which will be used as templates of old population (Bica \& Alloin 1987). In order to compare the spectra we first normalized them relative to the continuum at $5500 \AA$. A residual spectra was then defined as the difference between the normalized spectra of each galaxy $(G(\lambda) / G(55))$, and the normalized spectra of the elliptical galaxy NGC 3923 used as a template of old population $(O(\lambda) / O(55))$. In Fig. 3 we present the spectra of NGC 3923 and the residual spectra of NGC 3557 . Since both objects are representative of old population we can see that the residuals deviated less than $10 \%$ showing no systematic trends with wavelength.

However, when we examine the residuals of ellipticals in pairs we verify very clearly that the blue continuum of these objects, in the region around $4000-5000 \AA$, tend to be more intense than the template object. In Fig. 4 we present two extreme examples of early-type galaxies having large (g351a) and small (g362a) deviations in the blue region. In Fig. 5 we present the same plot for latetype galaxies (g222b and g266b). The steeper blue continuum can be used as a clear signature of young population (Bica \& Alloin 1986), which suggests that galaxies showing this effect have had recent star formation

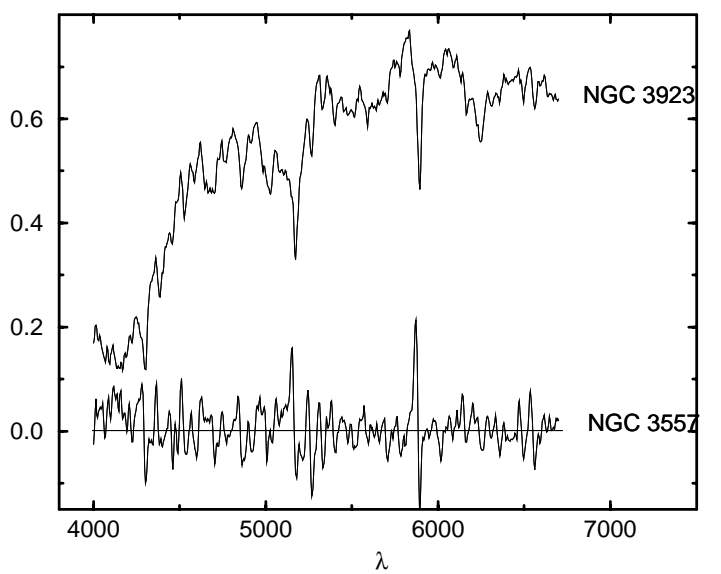

Fig. 3. Spectra of the field elliptical NGC 3923 used as a template for old stellar population. The residual spectra of the elliptical NGC 3557 show that both objects have a similar population

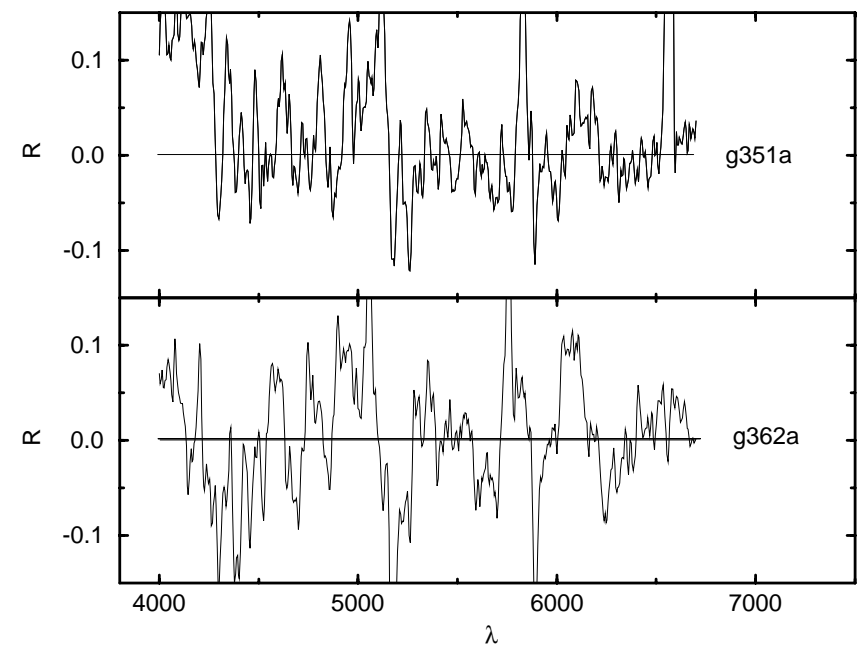

Fig. 4. Upper panel- residual spectra of g351a, an early type galaxy with blue excess. Bottom panel- early type galaxy with a residual spectra typical of an old population object

episodes. Using the residual of the normalized spectra $(R \lambda=G(\lambda) / G(55)-O(\lambda) / O(55))$ we can measure the extent of this effect by defining the residual $\left(R_{40}\right)$ evaluated at $4000 \AA$. As a first approximation we assumed that the spectral energy distribution of a galaxy is composed by the contribution of an old stellar population, similar to the adopted template object, $(O(\lambda))$ and a younger counterpart $(Y(\lambda))$. Using this approximation we obtain,

$R_{40}=\left(\frac{Y_{40}}{Y_{55}}-\frac{O_{40}}{O_{55}}\right) \frac{Y_{55}}{O_{55}+Y_{55}}$

where $\frac{Y_{40}}{Y_{55}}$ and $\frac{O_{40}}{O_{55}}$ are equal to the mean normalized flux of the young and old population, respectively. We can 
see from this result that the mean residual contains an information relative to the difference between the normalized spectra of the old and young population respectively. In Col. 13 of Table 1 we present the residual parameter measured for each galaxy in our sample. Actually, we can use the observations of Bica \& Alloin (1986) to verify that the young globular clusters of LMC with ages between $0.210^{7} \mathrm{yr}$ and $5010^{7} \mathrm{yr}$, and metallicities $-1.1 \leq Z / Z_{\odot} \leq-0.25$ have $1.42 \leq \frac{Y_{40}}{Y_{55}} \leq 2.27$. On the other hand, a typical old globular cluster have $0.63 \leq \frac{O_{40}}{O_{55}} \leq 0.68$, close to the ratio of 0.58 observed in NGC 3923. Therefore, the difference between the mean normalized flux of young and old population $\Delta=\frac{Y_{40}}{Y_{55}}-\frac{O_{40}}{O_{55}}$ is bounded to an interval limited by $0.74 \leq \Delta \leq 1.62$. Obviously the exact value of this parameter for a given object will depend on the mean metallicity and age of the assumed young and old population. However, this quantity have a typical value $\Delta \simeq 1.2 \pm 0.4$. Therefore, within the uncertainties due to the mettalicity and age history of the underlying population, the fiducial residual result to be approximately equal to the fractional contribution of the young stellar population flux at the reference wavelength $\left(R_{40} \simeq 1.2 \frac{Y_{55}}{O_{55}+Y_{55}}\right)$. On these grounds we can conclude from Fig. 4 that the young population contribution of g362a is probably less than $10 \%$, but it is substantially higher $(\simeq 15 \%)$ on g351a. For spirals the young stellar contribution is obviously higher as can be seen in Fig. 5 .

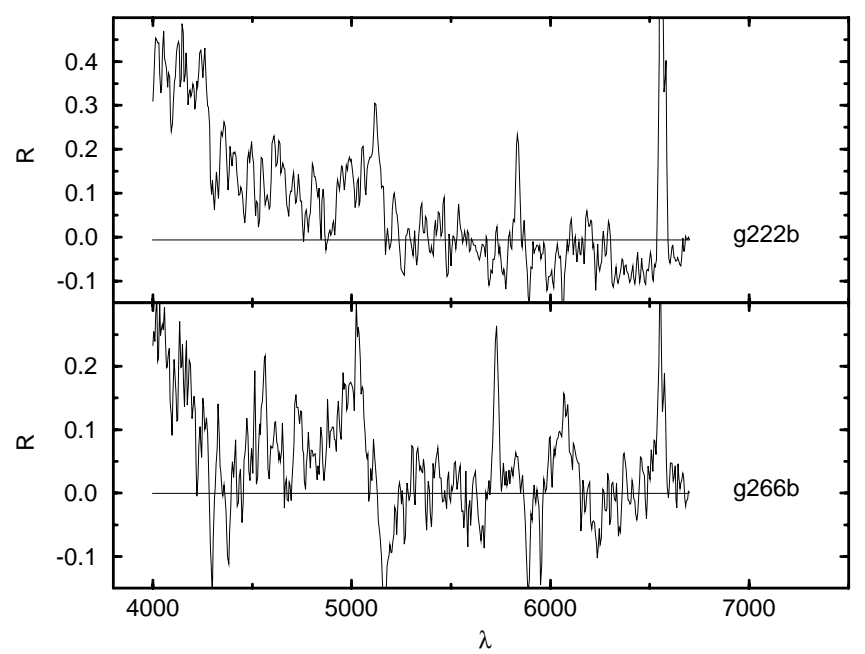

Fig. 5. Two examples of spirals showing a strong young population (g222b), and a moderate blue excess

Our spectra are representative of the central region of the objects, therefore we do not sample the same spatial dimensions in nearby and in the more distant objects. The slit aperture used in this work was 2.35 arcsec projected in the sky corresponding to an spatial region in the range $0.38-1.35 \mathrm{~h}^{-1} \mathrm{kpc}$, with a mean representative value of $0.84 \mathrm{~h}^{-1} \mathrm{kpc}$. Since there exist a gradient in surface brightness, and also in the star formation rate, in the central regions of interacting systems, the $R_{40}$ indicator might be affected by this aperture effect. Actually if we consider the $\mathrm{H} \alpha$ profiles of the list of strong interacting systems analysed by Bushouse (1987), in the same spatial region that we have sampled our objects, the flux drops by a factor of 10 . Since the profiles are roughly exponential we can predict that the mean $\mathrm{H} \alpha$ flux in the slit, for the nearest objects, should be approximately $86 \%$ of the central flux, while for the more distant it will drop to $59 \%$ of the central flux. Therefore we expect that one object, if observed in the same radial velocity range as our sample, would present a variation of the mean detected star forming rate of $45 \%$. To test for the presence of this effect we present in Fig. 6 a plot of $R_{40}$ as a function of the mean radial velocity of our pairs. We can observe that the high star forming region of this diagram is populated basically by nearby objects. However we caution that this is actually due to the Malmquist bias due to the limiting magnitude of our sample. In fact all the objects with $R_{40}$ higher than 0.35 are faint late type irregular spirals with mean absolute blue magnitude of $-18.0 \pm 0.8$, and therefore cannot be seen beyond $5000 \mathrm{~km} \mathrm{~s}^{-1}$ with apparent magnitude brighter than 15.5. For the brighter spirals we could not detect any systematic variation with distance. The absence of a strong aperture effect among spirals is probably related to the fact that our sample is not populated by highly interacting systems. As pointed out by Kennicutt et al. (1987) the contribution of the nuclear emission to the $\mathrm{H} \alpha$ luminosity, in a sample of paired galaxies similar to ours, amounts to $13 \%$ and is smaller than the one found on strongly interacting systems. For early type objects there is a correlation with radial velocity pointing the presence of an small systematic effect. For the low velocity objects $\left(V \leq 6000 \mathrm{~km} \mathrm{~s}^{-1}\right)$ the mean value of $R_{40}$ is $0.06 \pm 0.02$, while for the ones with higher velocity $R_{40}$ is $0.03 \pm 0.02$.

The residual parameter $R_{40}$ shows no clear correlation with the projected separation, normalized to the galaxy diameter, as can be seen from Fig. 7. For the early type objects there is a very marginal correlation $(r=0.20)$ in the sense that loose pairs tend to present a lower star forming rate. However, this is quite probably an spurious correlation resulting from a side effect of the slit aperture. As we mention before the residual parameter in more distant objects tend to be slightly smaller due to the larger spatial region sampled by the slit and the gradient on the star formation rate. On the other hand nearby wide separated pairs are far more difficult to detect due to chance projection of field galaxies along the line of sight. Therefore pairs of larger separation are more common at a higher distance and since in these objects the slit effect is larger an artificial correlation is introduced by this effect. In the case of spirals there is not even a marginal trend of the star formation with pair separation. The dominant effect is a larger dispersion of the residual parameter among objects 
of distinct morphological types. The average residuals of early-type objects in pairs is consistent with a young stellar contribution of the order of $0-10 \%$, while for spirals this contribution can be as large as $40 \%$, and even larger for late spirals. It is worthwhile mention that the star formation rate inferred from $\mathrm{CO}$ observations of a sample of binary galaxies (Combes et al. 1994) points to a weak but detectable correlation with component separation in the range Sep. $/ D_{25} \leq 10$. We remark that these two results are not contradictory since the sample of Combes et al. (1994) was richer in close interacting pairs having a higher level of tidally induced star formation rate.

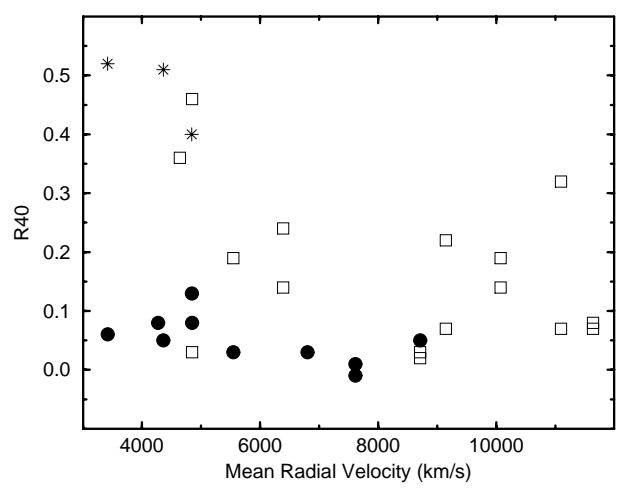

Fig. 6. Residual parameter, $R_{40}$, for early type objects $(\bullet)$, spirals $(\square)$ and irregulars $(*)$ showing no clear trend with the mean radial velocity

Far-infrared fluxes of the galaxies at 60 and $100 \mu \mathrm{m}$ were obtained by consulting the IPAC/IRAS Faint Source Catalog. A total of 7 objects out from the list of 17 confirmed binaries were detected. In 3 pairs (g280, g331, g363) the spatial resolution of IRAS was not enough to separate the emission of each member, and we assume that most of the emission comes from the spiral component in those cases. The mean infrared luminosity for these objects is $L_{\mathrm{FIR}} / L_{\mathrm{Blue}}=0.71 \pm 0.33$. Due to the presence of a larger number of late type spirals, the proportion of IRAS sources detected in the complete sample, described by Soares et al. (1995), is higher, 172 objects out from 189 pairs, when compared with the sample observed in the present work. After correcting for those cases where we could not separate the emission of each member we are left with 162 objects, and in this case we obtain $L_{\mathrm{FIR}} / L_{\mathrm{Blue}}=0.79 \pm 0.30$. These values can be compared with Xu \& Sulentic (1991) that have obtained $L_{\mathrm{FIR}} / L_{\mathrm{Blue}}=0.68 \pm 0.1$ for a list of SS binaries where both components are spirals, extracted from the sample of Karachentsev (1987) and $L_{\mathrm{FIR}} / L_{\text {Blue }}=0.50 \pm 0.1$, for a list of isolated field galaxies. Therefore we conclude that the FIR luminosities is also enhanced in our binary pairs when compared with field galaxies.
The absence of a clear correlation of $R_{40}$ with separation in a larger scale and the enhancement of FIR luminosities is probably related to the fact that even the small tidal interaction between wide separated pairs is enough to stimulate the mean star forming activity. Actually the detailed mechanism of the interaction depends on variables like the mass ratio and the coupling between spin and orbital angular moment, which are quantities that are almost impossible to be inferred for wide pairs. However, we notice that even galaxies with small residuals, like g362a, show an increase in the blue continuum not seen in the field objects like NGC 3557. Furthermore, the absorption lines in the $\mathrm{Mg}$ region ( $5175 \AA$ ) are much more intense, resulting in large negative values of the residuals. Therefore, galaxies in pairs are probably affected by either an increase in the star formation rate or a higher metallicity, when compared with objects in the field.

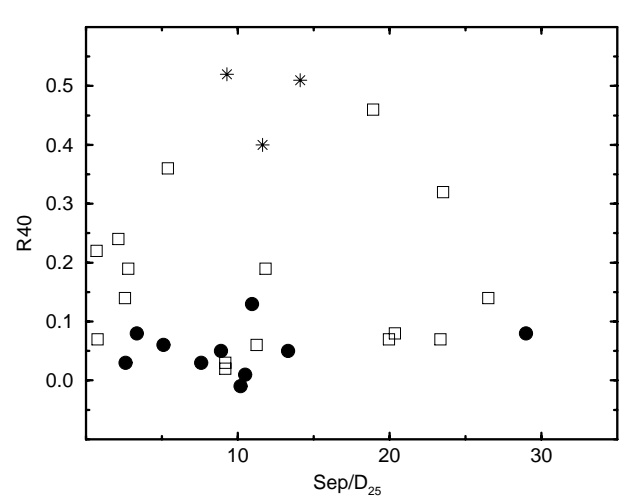

Fig. 7. Residual parameter, $R_{40}$, for early type objects $(\bullet)$, spirals $(\square)$ and irregulars $(*)$ showing no clear trend with the projected pair separation

\section{Conclusions}

The present sample was extracted from a list of probable binary galaxies determined through the use of a density enhancement criteria applied to the ESO-LV catalog (Soares et al. 1995). Our radial velocity measurements of this sample reveal that about $70 \%$ of the originally selected pairs are actually true binary systems. The sample contains a large fraction of pairs with separations larger than $100 \mathrm{kpc}$ and therefore can be useful to constrains the mass-to-light ratio since their mean separation is probably larger than the extent of their individual halos. It is our goal to measure more radial velocities in order to focus on that subject.

A comparison of the flux calibrated spectra with those of field ellipticals, used as template for old stellar population, shows that ellipticals in pairs tend to have a contribution $\simeq 5-10 \%$ of young stellar population. This 
result suggest that even the small interaction between galaxies belonging to loose pairs may be sufficient to increase the mean star formation rate at the required level. If large gaseous halos similar to the one reported by Hoffman et al. (1992) are a common feature among normal galaxies, then it may be possible that the small tidal field in loose pairs would be enough to disturb these halos that will become unstable and will increase the star formation rate. There is no correlation of the indicator of contribution of young stellar contribution with the projected pair separation, in agreement with CO observations.

Acknowledgements. We thank the referee P. Prugniel for helpful comments and useful suggestions. We are grateful to CTIO for the telescope time allocated to this project. We also acknowledge to $\mathrm{CNPq}$ and FAPESP for partial finnancial support to this project.

\section{References}

Athanassoula E., 1990, in Chemical and Dynamical Evolution of Galaxies, Franco J. and Mateucci F. (eds.). World Scientific Publ.

Baldwin J.A., Stone R.P.S., 1984, MNRAS 206, 241

Bica E., Alloin D., 1986, A\&A 162, 21

Bica E., Alloin D., 1987, A\&AS 70, 281

Bushouse H.A., 1987, ApJ 320, 49
Chengalur J.N., Salpeter E.E., Terzian Y., 1993, ApJ 419, 30

Combes F., Prugniel P., Rampazzo, Sulentic J.W., 1994, A\&A 281,725

Davis M., Huchra J., 1982, ApJ 254, 437

Horne K., 1986, PASP 98, 609

Hoffman G.L., Salpeter E.E., Lamphier C., Roos T., 1992, ApJ 388, L5

Karachentsev I.D., 1985, SvA 29, 243

Karachentsev I.D., 1987, Binary galaxies, Moscow, Nauka

Karachentsev I.D., 1990, in Paired and Interacting Galaxies, IAU Colloquium 124, Sulentic J.W., Keel W.C. \& Telesco C.M. (eds.), p. 3

Kennicutt R.C., Keel W.C., van der Hulst J.M., Hummel E., Roetttiger K.A., 1987, AJ 93, 1011

Kennicutt R.C., 1990, in Paired and Interacting Galaxies, IAU Colloquium 124, Sulentic J.W., Keel W.C. \& Telesco C.M. (eds.), p. 269

Lauberts A., Valentijn E.A., 1989, The Surface Photometry Catalogue of the ESO-Uppsala Galaxies, European Southern Observatory, Munich (ESO-LV)

Reduzzi L., Rampazzo R., 1995, Astrophy. Lett. Com. 30, 1-6

Reduzzi L., Rampazzo R., 1996, A\&AS 116, 515

Schombert J.M., Wallin J.F., Struck-Marcell C., 1990, AJ 99, 497

Soares D.S.L, de Souza R.E., de Carvalho R.R., Couto da Silva T.T., 1995, A\&AS 110, 371

Tonry J.L., Davis M., 1979, AJ 84, 1511

Toomre A., Toomre J., 1972, ApJ 178, 623

Xu C., Sulentic J.W., 1991, ApJ 374, 407 